\title{
Circuit model for a periodic array of slits sandwiched between two dielectric slabs
}

\author{
Raúl Rodríguez-Berral, ${ }^{1, a)}$ Francisco Mesa, ${ }^{1, b)}$ and Francisco Medina ${ }^{2, c)}$ \\ ${ }^{1}$ Department of Applied Physics I, University of Seville, 41012-Seville, Spain \\ ${ }^{2}$ Department of Electronics and Electromagnetism, University of Seville, 41012-Seville, Spain
}

(Received 22 January 2010; accepted 31 March 2010; published online 20 April 2010)

\begin{abstract}
This paper proposes an equivalent circuit model that uses lumped elements and transmission lines to explain the transmission of electromagnetic waves through a conducting screen periodically perforated with slits and sandwiched between two different dielectric slabs. The present model relies on the impedance-matching point of view, previously introduced by some of the authors, rather than on the surface plasmon polariton concept. Thus, the model constitutes a simple and insightful framework that easily leads to accurate qualitative and quantitative predictions about the nature of the transmission spectrum of such structures. (C) 2010 American Institute of Physics.

[doi:10.1063/1.3405721]
\end{abstract}

Extraordinary transmission has attracted much attention since its discovery. ${ }^{1}$ The plasmalike behavior of metals at optical frequencies and the excitation of surface plasmon polaritons (SPP's) were considered essential in a first stage. However it was soon evident that the same phenomenon could be scaled to much lower frequencies. ${ }^{2,3}$ In this latter case, the concept of spoof plasmons or SPP-Bloch waves ${ }^{4}$ rescued the theory that explains extraordinary transmission (ET) in terms of the excitation of surface waves. Nevertheless, ET through subwavelength apertures is also possible in some structures where SPP's cannot be defined. ${ }^{5-8}$ Recently, some of the authors have proposed a theory that puts emphasis on impedance matching rather than on the excitation of surfaces waves. ${ }^{9}$ This theory has been extended in Ref. 10 to the analysis of compound gratings ${ }^{11,12}$ and also accounts for SPP-free systems. ${ }^{6-8}$ All the above systems only involve perfect conductors and a uniform dielectric (free space). In practice, perforated metal screens are printed on supporting dielectric slabs, and it is clear that the addition of dielectric slabs creates additional opportunities for surface waves to propagate. Following the SPP theory, it allows for the appearance of additional transmission peaks in the spectrum. ${ }^{13}$ The aim of this contribution is to show that this situation can also be dealt with under the impedance matching paradigm. ${ }^{9,10}$ In order to focus our attention on the consequences of adding dielectric layers, we will concentrate on the study of relatively simple one-dimensional metallic transmission diffraction gratings. These structures have been thoroughly studied in the past ${ }^{14,15}$ and they are known to exhibit extraordinary transmission. ${ }^{16}$ Thus, a circuit model has been developed for metal strip gratings that provides an interesting physical insight on the nature of the phenomenon. As an additional advantage, it should be emphasized that the parameters of the equivalent circuit model can be determined with much less computational effort than that involved in SPP calculations, since only a few frequency points are required to reproduce the whole transmission spectrum.

\footnotetext{
a) Electronic mail: rrberral@us.es.

${ }^{\text {b) }}$ Electronic mail: mesa@us.es.

${ }^{c)}$ Electronic mail: medina@us.es.
}

Let us consider the structure shown in Fig. 1. The structure is excited by a plane wave at normal incidence and with the electric field oriented normally to the slits. Due to the symmetries of both the structure and the incident wave, the horizontal component of the electric field scattered by the screen is zero at the middle plane between adjacent slits. It implies that these middle planes can actually be considered as perfect electric walls (EWs). In this way the original problem is reduced to a parallel-plate waveguide problem with different dielectric regions plus an horizontal iris discontinuity (see Fig. 1). The propagation of the electromagnetic field along the different dielectric regions of the waveguide can be modeled, as usual in microwave engineering practice, ${ }^{17}$ using equivalent transmission lines (one for each launched mode).

In ET research, we are interested on the nondiffraction frequency region (no grating lobes), in such a way that only the transverse electromagnetic (TEM) mode propagates along the air-filled regions. However, the perforated screen (capacitive iris discontinuity) launches higher-order transverse magnetic (TM) modes, which can be modeled by means of the equivalent circuit shown in Fig. 2. The transmission lines at both sides of the discontinuity account for the propagation of the incident, transmitted, and reflected

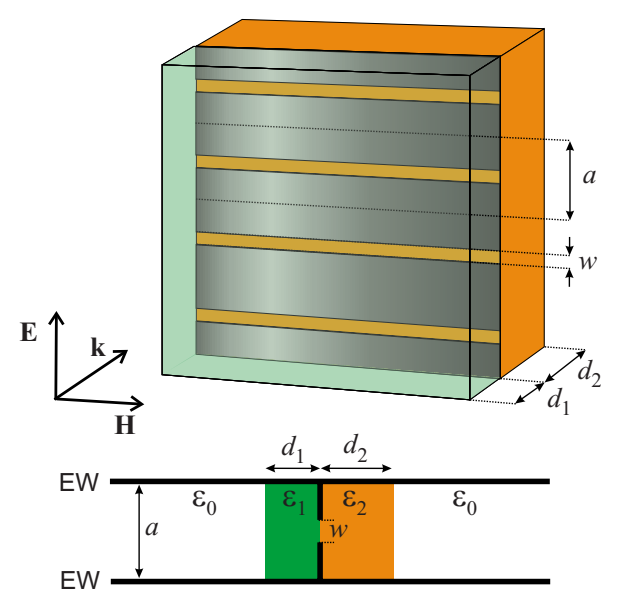

FIG. 1. (Color online) Structure under analysis (top) and equivalent waveguide problem (bottom). 


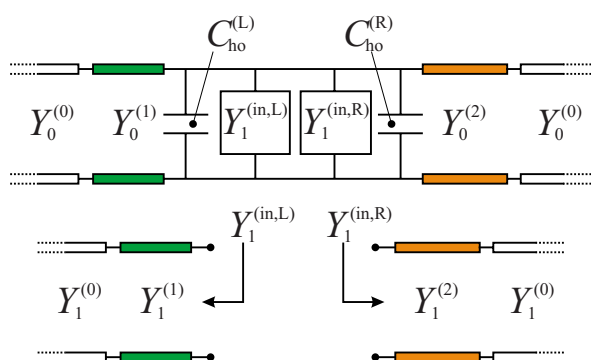

FIG. 2. (Color online) Transmission line and equivalent circuit model for the waveguide problem in Fig. 1.

TEM waves. Their characteristic admittances are given by the wave admittances in the corresponding dielectric media $\left(Y_{0}^{(0)}, Y_{0}^{(1)}\right.$, and $\left.Y_{0}^{(2)}\right)$. Higher-order TM modes are required to satisfy the boundary conditions in the screen and the aperture. Due to symmetry, only even-order TM modes are excited. Each of these higher-order modes are represented in the circuit by a shunt imaginary (reactive) admittance, which is given by

$$
Y_{n}^{\mathrm{in}, \mathrm{L} / \mathrm{R}}=A_{n}^{\mathrm{L} / \mathrm{R}} Y_{n}^{(1) /(2)} \frac{Y_{n}^{(0)}+\mathrm{j} Y_{n}^{(1) /(2)} \tan \left(\beta_{n}^{(1) /(2)} d_{1 / 2}\right)}{Y_{n}^{(1) /(2)}+\mathrm{j} Y_{n}^{(0)} \tan \left(\beta_{n}^{(1) /(2)} d_{1 / 2}\right)},
$$

where $A_{n}^{\mathrm{L} / \mathrm{R}}$ is a constant that accounts for the degree of excitation of the $\mathrm{TM}_{2 n}$ mode at the left/right hand side of the discontinuity and $\beta_{n}^{(1) /(2)}$ is the propagation wavenumber of the $\mathrm{TM}_{2 n}$ mode in the dielectric region (1)/(2). The characteristic admittances of the transmission lines are given by the following frequency-dependent wave admittances:

$$
Y_{n}^{(i)}=\frac{\sqrt{\varepsilon_{r i} \varepsilon_{0} / \mu_{0}}}{\sqrt{1-\left(f_{n}^{(i)} / f\right)^{2}}},
$$

where $f_{n}^{(i)}=n c /\left(\sqrt{\varepsilon_{r i}} a\right)$ is the cutoff frequency of the $\mathrm{TM}_{2 n}$ mode in the dielectric region with relative permittivity $\varepsilon_{r i}$ and $c$ denotes the speed of light in free space. As stated above, we are interested in the transmission spectrum of the structure for frequencies below the onset of the first grating lobe, which is launched at $f_{1}^{(0)}=c / a$ (the first grating lobe is related to the $\mathrm{TM}_{2}$ propagation in the surrounding free space). Characteristic admittances of highly evanescent modes $\left(f \ll f_{n}^{(i)}\right)$ are linear functions of frequency. Therefore, all of them can be accounted for by the global higher-order capacitances $C_{\mathrm{ho}}^{\mathrm{L} / \mathrm{R}}$. On the contrary, those TM modes that become relatively close to (or even above) their cutoff frequencies must be taken into account separately and are represented in the model by a shunt admittance whose frequency dependence is given by Eq. (1). In Fig. 2 we are assuming that only the first higher-order mode (the $\mathrm{TM}_{2}$ ) needs to be considered separately. This is enough to make qualitative predictions about the main features of the transmission spectrum. In the absence of dielectric slabs, all the higher-order modes are evanescent for frequencies up to the onset of the first grating lobe, $f_{1}^{(0)}$. The only salient effect is the total reflection of the impinging TEM mode at $f_{1}^{(0)}$ (Rayleigh-Wood anomaly). This fact is connected to the singular behavior of the admittance in (2) at such frequency. However, when the dielectric slabs are present, the first higher-order mode becomes propagative in the dielectric regions at frequencies lower than $f_{1}^{(0)}$. Consequently, the corresponding transmission line sections in Fig. 2 become propagative, thus allowing for a wide range of possible values of

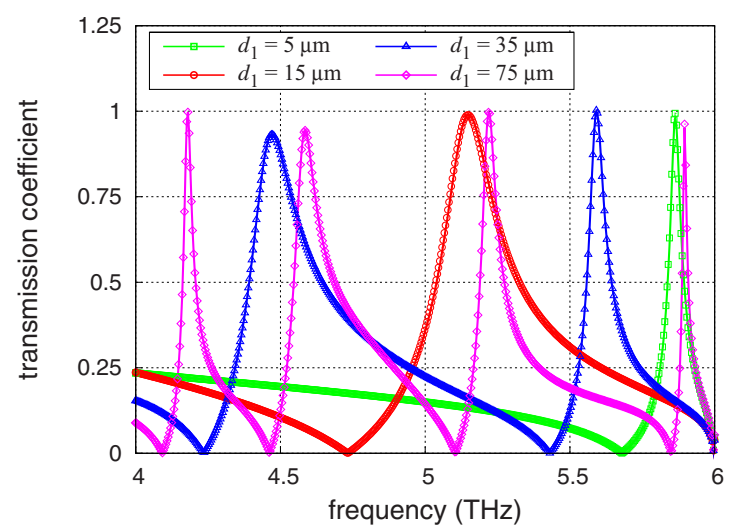

FIG. 3. (Color online) Transmission coefficient vs frequency for a periodic array of slits of width $w=5 \mu \mathrm{m}$ and period $a=50 \mu \mathrm{m}$ printed on a single dielectric slab $\left(d_{2}=0\right)$ of relative permittivity $\varepsilon_{r 1}=2.2$ and different thicknesses. Solid lines: data obtained with a mode matching code. Points: data obtained with our model.

the imaginary input admittances, $Y_{n}^{\mathrm{in}, \mathrm{L} / \mathrm{R}}$. This is the basic reason behind the rich phenomenology found in the transmission spectra of these structures.

In order to obtain numerical results, we first need to determine $C_{\mathrm{ho}}^{\mathrm{L}}+C_{\mathrm{ho}}^{\mathrm{R}}$, and the coefficients $A_{n}^{\mathrm{L} / \mathrm{R}}$. In general, the input admittances of all the modes above cutoff in each of the dielectric regions at the highest operation frequency must be included in the circuit model. In addition, it has been found that the inclusion of the evanescent mode with the lowest cutoff frequency is enough to obtain very accurate numerical results. This simple rule has been used to generate the data in this paper, which perfectly match full-wave results. The circuit parameters can be computed from the values of the reflection coefficient provided by some independent numerical code at just a few frequency values (we have used a home made full-wave mode matching code). For instance, if we consider two input admittances to the right and one to the left hand side of the discontinuity, we will have to compute four parameters (three $A_{n}$ coefficients plus the global higher-order capacitance). Four values of the reflection coefficient are then required. This is much faster than computing the whole spectrum using a full-wave approach for every frequency point and gives much more information than SPP theory, which only tell us about the critical frequencies where remarkable effects could be expected.

Let us consider a structure with a single dielectric slab. Figure 3 shows the transmission spectra for several dielectric substrates. The numerical results provided by our model have been obtained considering three input admittances (two at the dielectric side and one at the free-space side of the discontinuity) in all the cases shown in Fig. 3, which means that only four circuit parameters had to be determined. The agreement between our equivalent-circuit data and full-wave results is excellent. The presence of the usual transmission dip (Rayleigh-Wood's anomaly) at $f=c / a \approx 5.996 \mathrm{THz}$ is clear. For the two thinner slabs $(d=5$ and $15 \mu \mathrm{m})$ it is observed the appearance of an additional transmission dip at a lower frequency, and a transmission peak between the transmission dips. It is also observed that the additional transmission dip and the transmission peak appear at lower frequencies for $d=15 \mu \mathrm{m}$. These features can be easily predicted using our simple model. Since only one dielectric slab is present, one of the input admittances in Fig. 2, say $Y_{1}^{\mathrm{in}, \mathrm{L}}$, is simply proportional to the $\mathrm{TM}_{2}$ wave admittance in free 
space, which is given by Eq. (2) with $n=1$ and $\varepsilon_{r i}=1$. This admittance diverges at $f_{1}^{(0)}=c / a$, thus short-circuiting the line and causing a total reflection (Rayleigh-Wood's anomaly). Let us now focus on the remaining input admittance, $Y_{1}^{\mathrm{in}, \mathrm{R}}$, given by Eq. (1). For frequencies above the cutoff frequency of the $\mathrm{TM}_{2}$ mode in the dielectric region $\left[f_{1}^{(2)}=c /(a \sqrt{2.2})\right.$ $\approx 4.042 \mathrm{THz}]$, the mode is propagative in the dielectric region and its wave admittance $Y_{1}^{(2)}$ is real and positive. However, for frequencies below $f_{1}^{(0)}=c / a$, this mode is still evanescent in the free space region and its wave admittance $Y_{1}^{(0)}$ is imaginary and positive. Therefore, the two addends in the denominator of the input admittance are both real and have opposite sign. Taking also into account that the wave admittances $Y_{1}^{(2)}$ and $Y_{1}^{(0)}$ diverge at $f_{1}^{(2)}$ and $f_{1}^{(0)}$ respectively, there is necessarily an intermediate frequency value, $f_{\mathrm{R}}$, at which the denominator in Eq. (1) is zero. Therefore, the input admittance becomes infinite at $f_{\mathrm{R}}$, thus giving rise to the additional transmission dip. For frequencies below $f_{1}^{(0)}=c / a$, the wave admittance $Y_{1}^{(0)}$ is imaginary and positive (capacitive admittance). According to Eq. (1), the input admittance $Y_{1}^{\mathrm{in}, \mathrm{R}}$ is also capacitive at low frequencies but at $f=f_{\mathrm{R}}$ the sign of the denominator changes and the input admittance becomes inductive. Taking once again into account the divergence of the admittances at $f_{1}^{(0)}$ and $f_{\mathrm{R}}$, there is necessarily a frequency value $f_{\mathrm{T}}$ with $f_{\mathrm{R}}<f_{\mathrm{T}}<f_{1}^{(0)}$ at which the global capacitive admittance exactly compensates the inductive input admittance. Therefore, at $f=f_{\mathrm{T}}$ the circuit model constitutes a resonant $L C$ tank, which explains the transmission peak between transmission dips.

For the two thickest dielectric slabs considered in Fig. 3, it is clear that the above conditions for the additional transmission dip and the transmission peak are satisfied at lower frequencies, as observed in the plots. Furthermore, the tangents in Eq. (1) are periodic functions so that, for thick enough dielectrics, they can complete several cycles. It causes that the transmission spectrum presents several alternating transmission dips and peaks in the frequency range from $f_{1}^{(2)}$ to $f_{1}^{(0)}$, as observed for the $d=35$ and $75 \mu \mathrm{m}$ cases in Fig. 3.

For a screen sandwiched between two dielectric slabs, we will have two input admittances given by Eq. (1), and the above discussion applies to both of them. Therefore, if the dielectric slabs have different thicknesses and/or permittivities, there would be at least two transmission dips and two transmission maxima in the frequency range from $f_{1}^{(i)}$ to $f_{1}^{(0)}$, where $i$ corresponds to the dielectric with lower permittivity. In addition, a sandwiched screen would not present a transmission dip at the onset of the first grating lobe since the $Y_{1}^{(0)}$ admittance only appears in the model through the input admittances and these do not diverge at $f=f_{1}^{(0)}$. The above predicted facts are clearly confirmed by the curves shown in Fig. 4. In order to show the capabilities of the model, the slabs are chosen to be relatively thick, which results in a quite complicated transmission spectrum. In this case, we have considered two input admittances at each side of the

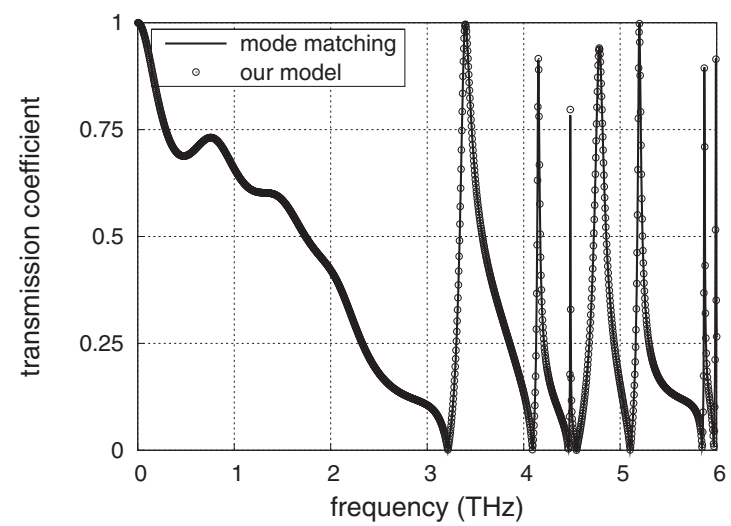

FIG. 4. Transmission coefficient vs frequency for a periodic array of slits of width $w=5 \mu \mathrm{m}$ and period $a=50 \mu \mathrm{m}$ sandwiched between two dielectric slabs with $\varepsilon_{r 1}=2.2, d_{1}=75 \mu \mathrm{m}, \varepsilon_{r 2}=4$, and $d_{2}=30 \mu \mathrm{m}$.

discontinuity (five circuit parameters). The agreement between the results generated with our model and those obtained with a full-wave mode-matching code is remarkable, especially taking into account the rather involved nature of the spectrum and the low number of circuit parameters needed.

The authors acknowledge the support of the Spanish Ministry of Science and Innovation, Spanish Junta de Andalucia and European Union FEDER funds (grants TEC2007-65376, CSD2008-00066 and TIC-4595).

${ }^{1}$ T. W. Ebbesen, H. J. Lezec, H. F. Ghaemi, T. Thio, and P. A. Wolff, Nature (London) 391, 667 (1998).

${ }^{2}$ M. Beruete, M. Sorolla, I. Campillo, J. S. Dolado, L. Martín-Moreno, J. Bravo-Abad, and F. J. García-Vidal, Opt. Lett. 29, 2500 (2004).

${ }^{3}$ M. Beruete, M. Sorolla, I. Campillo, J. S. Dolado, L. Martín-Moreno, J. Bravo-Abad, and F. J. García-Vidal, IEEE Trans. Antennas Propag. 53, 1897 (2005).

${ }^{4}$ J. B. Pendry, L. Martín-Moreno, and F. J. García-Vidal, Science 305, 847 (2004).

${ }^{5}$ E. Moreno, L. Martín-Moreno, and F. J. García-Vidal, J. Opt. A, Pure Appl. Opt. 8, S94 (2006)

${ }^{6}$ A. A. Kirilenko and A. O. Perov, IEEE Trans. Antennas Propag. 56, 3210 (2008).

${ }^{7}$ Y. Pang, A. N. Hone, P. P. M. So, and R. Gordon, Opt. Express 17, 4433 (2009).

${ }^{8}$ F. Medina, J. A. Ruiz-Cruz, F. Mesa, J. M. Rebollar, J. R. Montejo-Garai, and R. Marqués, Appl. Phys. Lett. 95, 071102 (2009).

${ }^{9}$ F. Medina, F. Mesa, and R. Marqués, IEEE Trans. Microwave Theory Tech. 56, 3108 (2008).

${ }^{10}$ F. Medina, F. Mesa, and D. C. Skigin, IEEE Trans. Microwave Theory Tech. 58, 105 (2010).

${ }^{11}$ D. C. Skigin and R. Depine, Phys. Rev. Lett. 95, 217402 (2005).

${ }^{12}$ M. Navarro-Cía, D. C. Skigin, M. Beruete, and M. Sorolla, Appl. Phys. Lett. 94, 091107 (2009).

${ }^{13}$ V. Lomakin and E. Michielssen, Phys. Rev. B 71, 235117 (2005).

${ }^{14}$ R. C. Compton, L. B. Whitbourn, and R. C. McPhedran, Int. J. Infrared Millim. Waves 4, 901 (1983).

${ }^{15}$ E. Popov, S. Enoch, G. Tayeb, M. Neviere, B. Gralak, and N. Bonod, Appl. Opt. 43, 999 (2004).

${ }^{16}$ J. A. Porto, F. J. García-Vidal, and J. B. Pendry, Phys. Rev. Lett. 83, 2845 (1999).

${ }^{17}$ R. E. Collin, Field Theory of Guided Waves (IEEE, Piscataway, New Jersey, 1991). 